\title{
Mental Health, Trust, and Robots: Towards Understanding How Mental Health Mediates Human- Automated System Trust and Reliance
}

\author{
Jordan R. Crawford, Ella-Mae Hubbard, Yee Mey Goh \\ Intelligent Automation, Loughborough University, Loughborough, Leicestershire, \\ United Kingdom, LE11 3GR \\ \{J.Crawford, E.Hubbard, Y.Goh\}@Lboro.ac.uk
}

\begin{abstract}
This paper proposes a conceptual model derived through current ongoing research that incorporates the potential relationship that mental health may have on trust and reliance calibration in automated systems (AS). Understanding the variables involved in the human-AS interaction allows system designers to better achieve trust calibration and avoid AS misuse and disuse. However, most of the research area is saturated with understanding how external and internal (both to the human) short-term cognitive symptoms mediate this critical relationship. Therefore, the present paper extends human-AS trust literature with common mental disorders (CMDs) as outlined by the Adult Psychiatric Morbidity Survey (APMS) and incorporates them into existing models within the engineering and psychology subject areas to begin to understand what this relationship may look like. It is hoped that this paper will expand the scope of human factors (specifically human-AS trust) to include mental disorders.
\end{abstract}

Keywords: Mental Health · Common Mental Disorders · Automated Systems · Trust $\cdot$ Reliance

\section{$1 \quad$ Introduction}

As technology evolves, it becomes more a part of an individual's life. Whether they are needed to work alongside it at work, travel home in it or relax at home using it, people are increasingly required to co-exist with automated systems (AS) growing in their reach and capabilities. As humans assume an overseer role [1]-[3], the relationship between human and machine is becoming just as important as the interpersonal one always has been. This paper discusses mental health in this context in order to begin to understand its importance in ensuring the fruitfulness of the human-AS relationship.

In order for AS to be successful in its deployment, it is widely considered that it has to be trusted to be fully adopted by the end user(s), or it will not be used to its fullest potential [4]. Though in more detail, individuals must appropriately calibrate their trust to form the correct level of system reliance in order to avoid detrimental cost to the system. AS misuse and disuse, as introduced by Parasuraman \& Riley [3] as excessive underreliance and overreliance respectively, have had prevalent examples in personal and non-personal navigation [5], [6] due to the increased access 
to satellite technology. Therefore, it is essential that researchers understand humanAS reliance decisions in as much detail that is possible in order to improve these reliance decisions. While past research has focused on cognitive decision-making factors in this relationship [7]-[9], it has begun to move towards more affective consideration [1], [10]. However, this paper proposes that a deeper look into human psychology is needed at the potential sources of undesirable cognitive and affective variation in this area. In this case, individual quality of mental health. This project incorporates mental health factors into conceptual human-AS trust and reliance models to better understand the varying and complex research in this area.

According to the Adult Psychiatric Morbidity Survey (APMS) [11] carried out in 2014 (conducted every seven years by the NHS), one in six $(17 \%)$ of the working age population of Great Britain meet diagnostic criteria for a common mental disorder (CMD). The most common of these issues are anxiety, depression or a combination of the two [11], though there is an extensive spectrum of distinct diagnoses which result in different symptoms and require different approaches of care. While causing emotional distress and interference with day-to-day life, CMD's do not usually affect insight or cognition [11]. Due to its sizable portion of the working population (Fig. 1) resulting in the cumulative cost of CMD's to be larger than other psychiatric disorders, the present working paper presents interest in incorporating all CMD diagnoses deemed appropriate by the APMS [11].

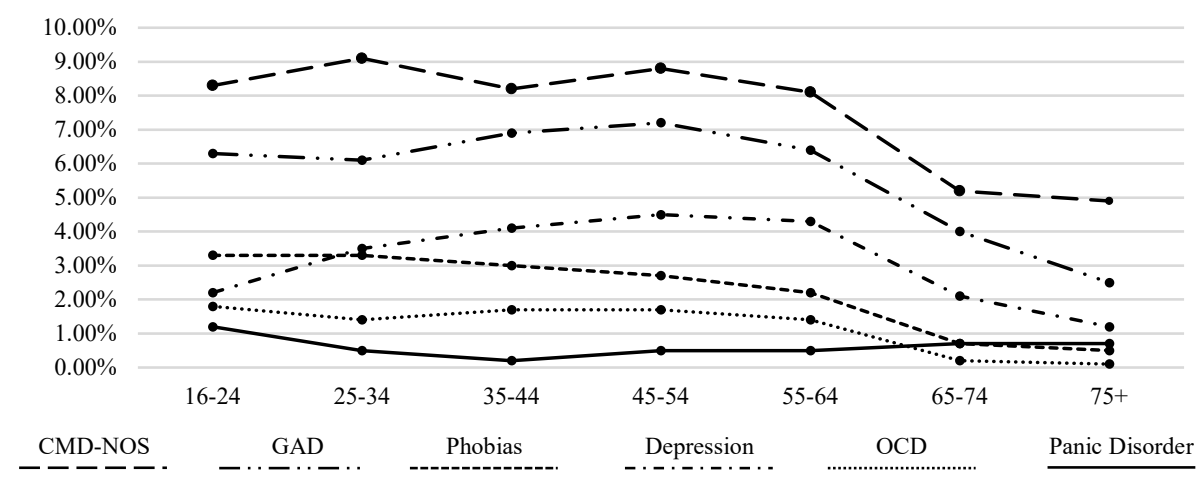

Fig. 1. APMS prevalence of CMD by age $[12$, Fig. $1 \mathrm{~b}]$ shows greater pervasiveness in the working age (16-64). GAD, Phobias, Depressive Episode, OCD, and Panic Disorder are excluded from CMD-NOS.

APMS classifications, employed by the Mental Health Foundation [12], follow an amalgamated classification of the non-psychotic diagnoses and symptoms in the World Health Organization $10^{\text {th }}$ International Classification of Disease (ICD-10) [13]. These include generalised anxiety disorder (GAD), depression, phobias, obsessivecompulsive disorder (OCD), panic disorder, and those common mental disorders that are not otherwise specified (CMD-NOS). It should be noted that 'CMD-NOS' is defined as individuals showing adequate symptom criteria to be considered a mental 
health disorder with 'mixed-anxiety and depression', but fell short of the criteria for any specific CMD [11]. Therefore, it follows that individuals with this diagnosis therefore cannot be classed as having any other CMD, whereas the other five disorders (with the exception of panic disorder and phobias which have mutually exclusive diagnostic criteria [13]) can appear in more than one case.

\section{The Model}

The conceptual model proposed (Fig. 2) and the following explanations of the types of trust is developed from the model of trust and reliance in AS by Hoff \& Bashir [7], in the review that considers all empirical research regarding human-AS trust and reliance. This model is similar to those proposed in other reviews and meta-analyses [8], [9] though is visually more effective than its counterparts due to its detailed inclusion of knowledge within a single intuitive model ideal for the purpose of this paper. The model considers trust in three parts, dispositional trust, situational trust, and learned trust [7].

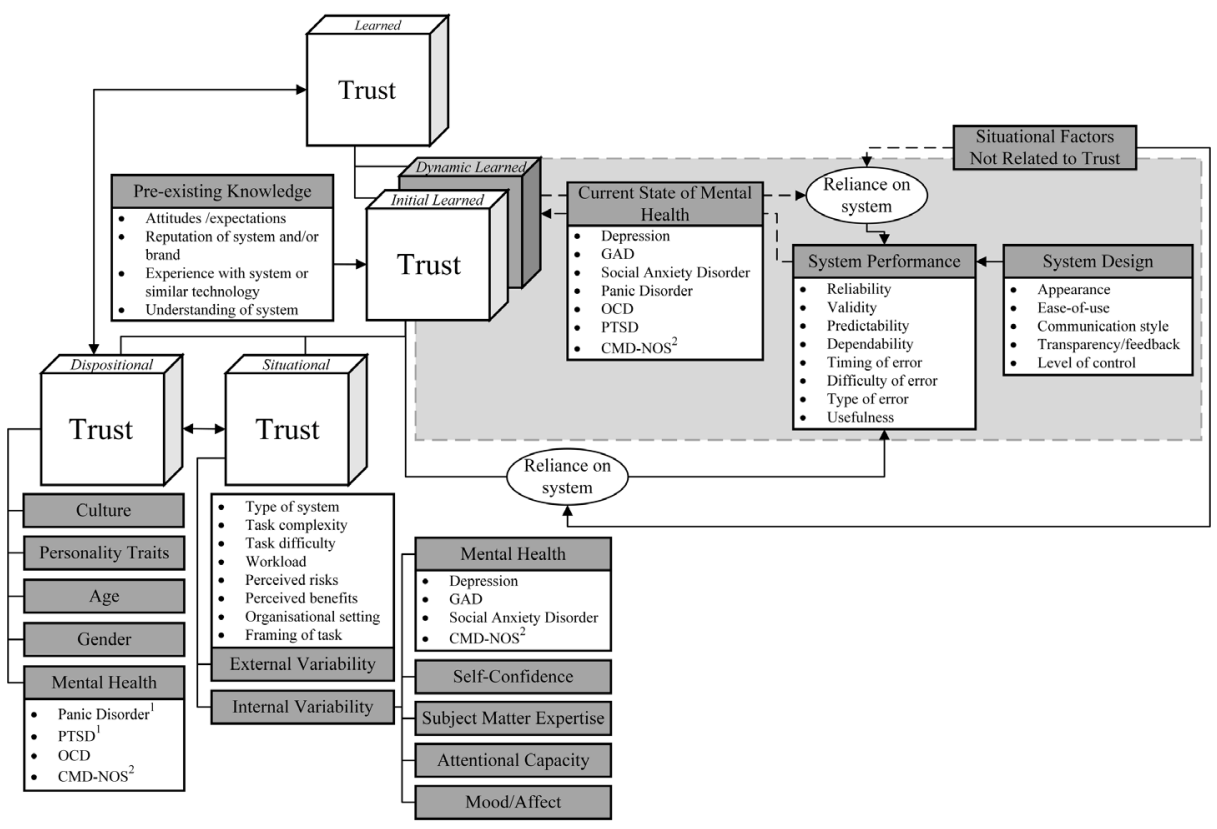

Fig. 2. Conceptual model developed from Hoff \& Bashir [7]. Within a single interaction, the shaded area depicts processes taking place and dashed arrows are elements that can change [7].

\footnotetext{
${ }^{1}$ While meeting criteria for its designated category, these factors do not hold exclusive symptoms to its designated category.

${ }^{2} \mathrm{CMD}-\mathrm{NOS}$ is depicted in the proposed model in all types of trust since as defined as a catch-all mixed-anxiety and depression mental disorder [11], it is possible that it is both dispositional and situational whilst also influencing dynamic trust.
} 
Dispositional trust is concerned with an individual's overall tendency to trust AS, independent of context or a specific system, as categorised by culture, age, gender, and personality traits. Dispositional trust factors are influenced by long-term tendencies arising from biology and the environment the individual is subjected to. As a result, these factors are less likely to change over time than those trust moderators of situational and learned trust. They can also alter trust formation in any situation, and therefore are not to be confused then with certain characteristics such as self-confidence, which is context dependent.

Situational trust concerns us with the context of the interaction between an individual and the AS. Fig. 2 shows that situational trust involves both external environmental factors and the internal mental state of the individual. Therefore, different to personality traits in dispositional trust, internal variability fluctuates depending on their current mental state or their expertise.

Learned trust concerns us with attitudes that are gained from past experiences relevant to a specific AS. This factor of trust is directly influenced by the individuals pre-existing knowledge of the system and the AS's performance, perceptions of the latter influenced by system design. Note dynamic learned trust in Fig. 2, since during an individual's interaction with an AS, their trust and reliance strategy may dynamically fluctuate to reflect their reaction to the system's real-time performance.

This model suggests that dispositional trust, situational trust and initial learned trust form the initial reliance strategy that an individual enters a relationship with an AS. This initial reliance strategy, along with system design are the two sources that dictate system performance. System performance is then recognised by the individual in their real time dynamic learned trust, which, along with situational factors not related to trust, then develops reliance on the particular system. Also following Hoff \& Bashir [7], Understanding that, as outlined by Lee \& See "trust guides-but does not completely determine — reliance” [4, pp. 51], moderators that influence reliance but do not have any relationship to trust, such as situational awareness [9] and time constraints [14] to name a couple are included. Such that these factors may have a mediating effect of reliance behavior, but do not have effect on human trust. For example, Endsley [9] considers situational awareness to be a moderator of the human-AS interaction performance but not of AS trust.

This working paper considers the influence of CMDs on human-AS trust and reliance under each of the different categories of human-AS trust, dispositional, situational, and learned as proposed in the model presented. More specifically, CMDs as outlined throughout this paper and as shown in fig. 1 are considered to mediate trust and reliance within dispositional trust, situational trust (internal variability) and as a filter within the dynamic learned trust and reliance relationship. While initial learned trust may have an indirect influence on how mental health mediates human-AS trust and reliance behavior through dictating an individual's initial reliance strategy, it does not include specific mental health factors itself. This is because the initial learned trust node describes purely cognitive factors associated with a particular system [7]. This includes attitudes/expectations, knowledge of the system's reputation, experience with the system (or a similar technology), and understanding of the system, and CMDs do not usually affect insight or cognition [11]. 


\subsection{Dispositional Trust}

CMDs as identified by the APMS [11] can be organised into dispositional consideration consisting of panic disorder, PTSD, OCD and CMD-NOS each for their own unique reason. Note that the satisfaction criteria for dispositional factors as defined by Hoff \& Bashir [7] is that they must both alter trust in every situation and be relatively stable over time. While these criteria when approaching factors such as age and gender are simple to decide upon, when applying them to mental conditions they become somewhat ambiguous. Therefore, these can be defined as possessing symptoms that remain consistent and are non-fluctuating from the point of the individual's conception of CMD of which may also alter trust in every situation. The ICD-10 [13] is utilised to define the following diagnosis criteria used.

Panic disorder diagnosis requires the inclusion of persistent worry in anticipation of an anxiety attack in which is present in all aspects of an individual's life. This may also include a consistent change in behaviour after conception of the disorder in comparison to their behaviour beforehand. However, while this qualifies panic disorder as a dispositional factor, a key symptom of panic disorder is panic attacks. Panic attacks occur irregularly and unexpectedly, sometimes through environmental triggers but often what appears to be random. The former criteria however are the distinction between an individual who suffers with panic disorder rather than one in which suffers only with panic attacks, regardless of the quantity or frequency. Recommendations for future human-AS trust and reliance research include testing the unique symptoms of panic disorder, namely the effect of consistent negative anticipation.

Post-traumatic stress disorder (PTSD), which may last up to an individual's entire life from its conception, has a diagnosis that requires the inclusion of persistent subjection to two or more of the following: difficulty falling asleep, emotional detachment, anhedonia, irritability or outbursts of anger, difficulty in concentrating, hypervigilance, or an exaggerated startle response. As a result, these symptoms satisfy the criteria to be considered dispositional. However, similar to panic disorder, PTSD is popularly categorised as a reaction to intrusive flashbacks, memories, or dreams regarding an experienced distressing event, which is inconsistent. Also, PTSD is sometimes categorised by its subject's avoidance of specific scenarios, which therefore does not satisfy dispositional criteria. Therefore, an analysis of the individual's personal experience with their PTSD is essential to understand its impact with AS on a case by case basis. For example, interesting future research may analyse the specific effect of PTSD as a result of a distressing experience with an AS, or otherwise test the dispositional symptoms of PTSD the subject possesses. Within general psychology literature, studies have found that those diagnosed with PTSD have an increased and persistent tendency to distrust, even towards those that are close to them [15]. Therefore, with research showing that human-AS interactions are highly comparable to those which are interpersonal [16], future directions in this area may identify whether this holds true with PTSD.

Obsessive-compulsive disorder $(O C D)$ is characterised as a combination of recurrent and persistent obsessions (thoughts, ideas, or images) and compulsions (acts). It is an essential part of OCD that the individual understands the symptoms are excessive or unreasonable, they are unpleasant, and they are unsuccessfully resisted. As a result, carrying out their compulsions are not pleasurable but are followed with 
temporary relief of the tension or anxiety that drove the compulsion. OCD therefore causes a large amount of distress and interferes consistently with individual and social life, usually though distraction and/or wasting time. Therefore, future research may benefit from testing these symptoms as a part of OCD. This is because, for example, while distractions have been identified to have a mediating effect in the trust $\&$ reliance relationship in some cases [17], [18], they did not in an automated collision warning system task [19] of which the reason for this has not been definitively explained. However, OCD distractions are more extreme and unavoidable for those diagnosed with the disorder [13] to even what would appear to be minor problems to someone that does not suffer from it. Thus, outlining the potential for interesting comparative research in this area.

\subsection{Situational Trust}

CMDs as identified by the APMS [11] can be organised into situational consideration (internal to the individual) consisting of Depression, GAD, Social Anxiety Disorder and CMD-NOS. Internally variable traits can be seen as an extension of dispositional traits, in that while individuals have more enduring traits that arise as dispositional moderators, individuals also exhibit more impermanent traits that vary depending on any number of contextual, situational or time-related reasons. Identical to dispositional trust, The ICD-10 [13] is utilised to define the following diagnosis criteria used.

Depression is one of two core mental disorder sources, the other being anxiety [11], that may fluctuate in any one person from day-to-day. The other CMDs defined are on the spectrum of these two and as a result suffer from either depressive or anxiety symptoms. It is important to note that while individuals may have both depression and another CMD simultaneously, depression in this case is deliberately separated by the APMS to the other CMDs and considered its own disorder if not better explained by another CMD [13]. At this point, there has been research to suggest that depression is negatively correlated with interpersonal trust [20]. Therefore, if interpersonal and human-AS interactions are as similar as research suggests that they are [16], work is needed to identify if depression in this case holds consistent.

Generalised anxiety disorder $(G A D)$ is separated from anxiety as a symptom and is diagnosed through prolonged feelings of tension, feelings of apprehension and worry about every-day scenarios. Common symptoms include a heightened startle response, difficulty concentrating, intense worry, persistent irritability, and difficulty sleeping. However, GAD is unstable and therefore is not hypothesised as a consistent moderator of human-AS trust, even for the same individual on different days. It does however, as its name suggests, permeate generally through all environmental circumstances. There has been some relevant research tackling some of the symptoms associated with GAD, such as lack of sleep [21]. However, considering GAD is the most prevalent CMD (particularly in the working age range as shown in Fig.1), research is needed to assess the mental disorder in its entirety rather than its symptoms in isolation in order to understand the effects of them in combination. GAD symptoms such as feelings of apprehension, heightened startle response, worry, and irritability are all topics which may have an influential role in reactions and collaboration with imperfect AS.

Social anxiety disorder, or social phobias is predominantly the fear of scrutiny in comparatively smaller groups to large crowds (which distinguishes it from 
agoraphobia) and are usually associated with low self-esteem and a fear of criticism. This may result in the disorder being discrete, in that it involves the fear and avoidance of specific social situations unique to the individual subject or diffuse into almost all social situations outside of those which are already familiar. Aside from actively avoiding and not participating in certain social activities and interaction (including eye-contact in some cases), social anxiety disorder also results in symptoms such as hand tremors, nausea, or urgency of micturition. Human-AS interaction literature would therefore do well to assess social anxiety disorder and its common theme of avoidance of interaction and whether this applies to human-AS interaction, specifically if the symptoms influence trusting intentions and reliance behaviour. Human-AS collaboration (as opposed to human-human collaboration) may have many benefits for those that suffer from social anxiety due to the potential of creating AS with an absence of judgement and social pressure, such as eye contact and conversation. Also, an examination of discrete case by case examples versus individuals that have a diffused diagnosis would be interesting to understand the differences between the two in the context of human-AS trust and reliance.

\subsection{Dynamic Learned}

Learned trust is trust that is relative to characteristics of the AS drawn from an operator's evaluations of a system drawn from past experience or the current interaction [7]. Further, dynamic learned trust, being one of the interests of this paper, considers trust which changes over the course of an individual's interaction with an AS as a reaction to its real-time perceived performance [7]. It therefore identifies factors that influence how mental health may impact trust and reliance beliefs, attitudes, intentions, and behaviours [4], [22], [23]. The hypothesised dynamic learned trust relationship may be similar to Tomlinson \& Mayer's model of trust repair [24], which incorporates Weiner's [25] attribution theory and Mayer et al.'s [26] feedback loop, where individuals evaluate whether their experience was positive or negative, influencing the way the individual feels, their expectations, and their behaviour going forward.

In order to avoid AS misuse and disuse, an equilibrium of reliance settling in the middle of these is the aim of any system design and implementation [4]. The conceptual model (Fig. 1) presented is effective at describing the importance of appropriate reliance calibration. This is because it can be considered that an individual's reliance strategy to be a function of their initial reliance strategy, generated from their initial trust, and their actual reliance on the AS, generated from their dynamic learned trust [7]. This identifies a key caveat to real time reliance in the form of misuse and disuse. In turn of system performance determining an individual's dynamic learned trust and therefore reliance strategy, this resulting reliance strategy may have a real time impact on perceived or even actual system performance caused by a resulting misuse or disuse [27]. This may logically result in the individual distrusting an AS more due to its poor performance, leading to the individual using the system less, resulting in the humanAS relationship being compromised and thus further poor performance due to the decreasing quality of collaboration. As a result, the interaction runs the risk of perpetuating and amplifying poor or excessive trust and reliance, highlighting the need to calibrate trust and reliance first time. 
Further research will investigate the relationship between system performance and dynamic learned trust, and dynamic learned trust and reliance on the system respectively passing through a 'filter' of the individual's current state of mental health in the present interaction. This will follow the suggestion of models such as that proposed by Tomlinson \& Mayer [24] in general trust psychology which identifies that positive and negative outcomes form affective emotional reactions, which in turn generate their response and future reliance intentions. Outcome based dynamic trust models have since appeared in human-AS trust literature [8], [9] but fail to include emotional cognitive processes. Thus Fig. 3 shown is a conceptual model of this relationship. This paper proposes that since research is starting to find that affective factors mediate trust and reliance throughout the human-AS interaction [1], [10], mental health factors that have more complex and extreme symptoms than solely affect (such as positive/negative mood) have an mediating effect in their own right. While CMDs do not usually affect insight and cognition [11], mental health is proposed to have a reactive or distractive mediation to AS performance.

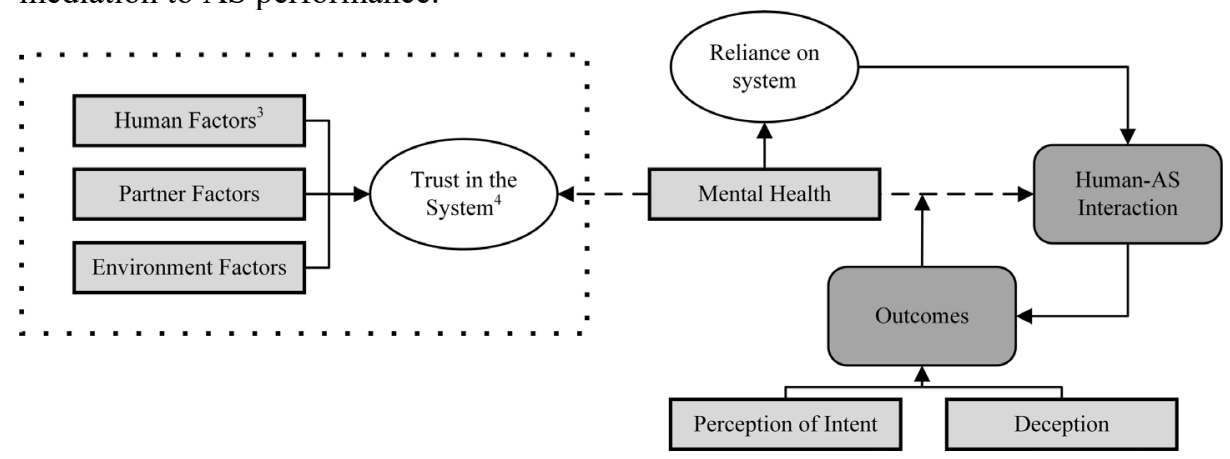

Fig. 3. A conceptual organization of trust influences highlighting trust development (adapted from [8, Fig. 1]). The dotted box represents the trust development part of the process and the dashed line represents trust calibration over time.

\section{Conclusion}

If mental health mediates the human-AS trust relationship, current approaches to holistic system design are incomplete. Therefore, absence of consideration of such is likely to have a detrimental system cost due to the loss of calibration in trust and reliance, resulting in either misuse or disuse of AS [3]. During collaborative tasks of any nature, this relationship is then undermined. Further, with the large presence of CMDs within the working population (Fig.1), the absence of consideration is also likely to have a high cumulative cost above all other mental health conditions [11], highlighting the need to prioritise the factors proposed within this paper. Following cognitive and affective literature, understanding mental health moderators brings us

\footnotetext{
${ }^{3}$ Includes propensity to trust

${ }^{4}$ Represents trust at a specific point in the human-AS interaction
} 
closer to understanding how humans are approaching the robotic future, and thus providing this future with priorities to ensure human flourishing.

The proposed model is considered conceptual, therefore research into these indicators is either rare or non-existent at the time of writing. As a result, some may have little effect. Contrastingly, others may have critical influential effects to individual trust and reliance (and therefore human-AS collaborative performance) that have otherwise been greatly neglected.

This conceptual model is a part of an ongoing research project of which is currently finalizing empirical evidence into GAD and Depression, though other factors described are largely unexplored.

\section{References}

1. Merritt, S.M.: Affective Processes in Human-Automation Interactions. Hum. Factors. 53, 356--370 (2011)

2. Lee, J.D., Moray, N.: Trust, Self-confidence, and Operators' Adaptation to Automation. Int. J. Hum. - Comput. Stud. 40, 153--184 (1994)

3. Parasuraman, R., Riley, V.: Humans and Automation : Use, Misuse, Disuse, Abuse. Hum. Factors. 39, 230--253 (1997)

4. Lee, J.D., See, K.A.: Trust in Automation: Designing for Appropriate Reliance. Hum. Factors. 46, 50--80 (2004)

5. Speake, J., Axon, S.: "I Never Use 'Maps' Anymore”: Engaging with Sat Nav Technologies and the Implications for Cartographic Literacy and Spatial Awareness. Cartogr. J. 49, 326-$335(2012)$

6. The Nautical Institute: Official Report No. 7042OR: Grounding of Cruise Ship (2002)

7. Hoff, K.A., Bashir, M.: Trust in Automation: Integrating Empirical Evidence on Factors That Influence Trust. Hum. Factors. 57, 407--434 (2015)

8. Schaefer, K.E., Chen, J.Y.C., Szalma, J.L., Hancock, P.A.: A Meta-Analysis of Factors Influencing the Development of Trust in Automation: Implications for Understanding Autonomy in Future Systems. Hum. Factors. 58, 377--400 (2016)

9. Endsley, M.R.: From Here to Autonomy: Lessons Learned from Human-Automation Research. Hum. Factors. 59, 5--27 (2017)

10.Stokes, C.K., Lyons, J.B., Littlejohn, K., Natarian, J., Case, E., Speranza, N.: Accounting for the Human in Cyberspace: Effects of Mood on Trust in Automation. In: Proceedings of the IEEE International Symposium on Collaborative Technologies and Systems, pp. 180-187. IEEE (2010)

11.McManus, S., Bebbington, P., Jenkins, R. Technical report, NHS Digital (2014)

12.Mental Health Foundation: Fundamental Facts About Mental Health. Technical report, Mental Health Foundation (2016)

13.World Health Organisation: The ICD-10 Classification of Mental and Behavioural Disorders: Clinical Descriptions and Diagnostic Guidelines. Technical report, World Health Organisation (1992)

14.Rice, S., Keller, D.: Automation Reliance Under Time Pressure. Cogn. Technol. 14, 36--44 (2009)

15.Cias C.M., Young, R., Barreira, P.: Loss Of Trust: Correlates Of The Comorbidity Of Ptsd and Severe Mental Illness. J. Pers. Interpers. Loss. 5, 103--123 (2009)

16.Madhavan, P., Wiegmann, D.A.: Similarities and Differences Between Human-Human and Human-Automation Trust: An Integrative Review. J Theor. Issues Ergon. Sci. 8, 277--301 (2007) 
17.Wickens, C.D.: Multiple resources and performance prediction. J Theor. Issues Ergon. Sci. 3, 159--177 (2002)

18.Phillips, R.R., Madhavan, P.: The Effect of Distractor Modality and Processing Code on Human-Automation Interaction. Cogn. Technol. Work. 13, 233--244 (2011)

19.Lees, M.N., Lee, J.D.: The Influence of Distraction and Driving Context on Driver Response to Imperfect Collision Warning Systems. Ergonomics, 50, 1264--1286 (2007)

20.Schneider, I.K., Konjin, E.A., Righetti, F., Rusbult, C.E.: A Healthy Dose of Trust: The Relationship Between Interpersonal Trust and Health. Pers. Relatsh., 18, 668--676 (2011)

21.Reichenbach, J., Onnasch, L., Manzey, D.: Human Performance Consequences of Automated Decision Aids in States of Sleep Loss. Hum. Factors. 53, 717--728 (2011)

22.Ajzen, I., Fishbein, M.: Understanding Attitudes and Predicting Social Behaviour. Prentice Hall, New Jersey (1980)

23.Fishbein, M., Ajzen, I.: Belief, Attitude, Intention and Behavior. Addison Wesley, Reading (1975)

24.Tomlinson, E.C., Mayer, R.C.: The Role of Causal Attribution Dimensions in Trust Repair. Acad. Manag. Rev. 34, 85--104 (2009)

25.Weiner, B.: An Attributional Theory of Achievement Motivation and Emotion. Psychol. Rev. 92, 548--573 (1985)

26.Mayer, R.C., Davis, J.H., Schoorman, D.F.: An Integrative Model of Organizational Trust. Acad. Manag. Rev. 20, 709--734 (1995)

27.Manzey, D., Bahner, J.E., Hueper, A.: Misuse of Automated Aids in Process Control:

Complacency, Automation Bias and Possible Training Interventions. In: Proceedings of the Human Factors and Ergonomics Society 50th Annual Meeting, pp. 220-224. (2006) 